\title{
Crystal structure of (E)-2-(3,6-bis(diethylamino)-9 $\mathrm{H}$-xanthen-9-yl)- $\mathrm{N}^{\prime}$-(quinoxalin-2-ylmethylene) benzohydrazide, $\mathrm{C}_{37} \mathrm{H}_{36} \mathrm{~N}_{6} \mathrm{O}_{2}$
}

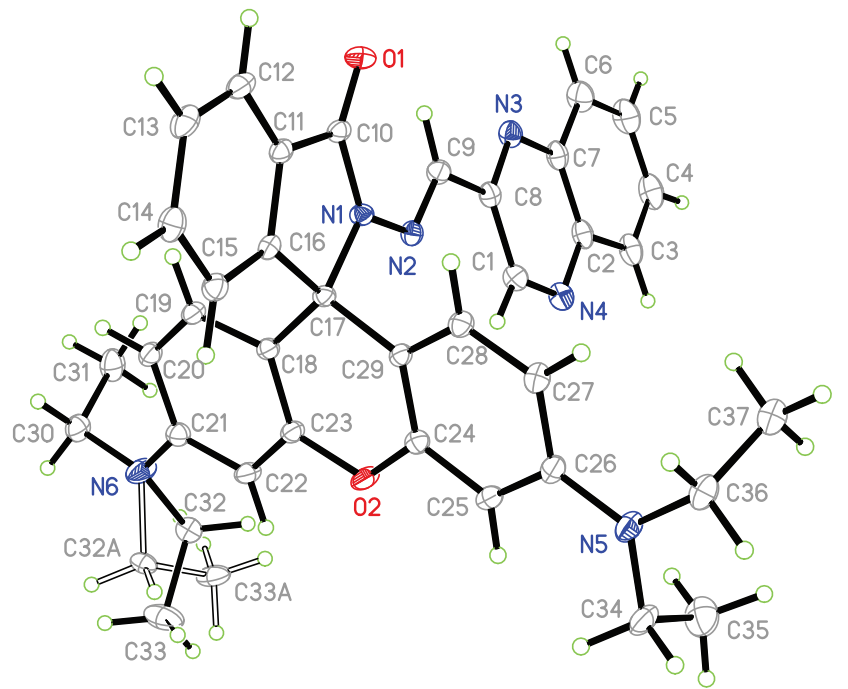

https://doi.org/10.1515/ncrs-2019-0290

Received April 22, 2019; accepted June 20, 2019; available online September 9, 2019

\section{Abstract \\ $\mathrm{C}_{37} \mathrm{H}_{36} \mathrm{~N}_{6} \mathrm{O}_{2}$, monoclinic, $P 2_{1} / c$ (no. 14), $a=9.18664(12) \AA$, $b=14.50209(16) \AA, c=23.1787$ (3) $\AA, \beta=94.0829(12)^{\circ}, Z=4$, $V=3080.16(7) \AA^{3}, \quad R_{\mathrm{gt}}(F)=0.0398, \quad w R_{\mathrm{ref}}\left(F^{2}\right)=0.1041$, $T=113.5(4) \mathrm{K}$.}

\section{CCDC no.: 1935393}

The asymmetric unit of the molecular structure is shown in the figure. Table 1 contains crystallographic data and Table 2 contains the list of the atoms including atomic coordinates and displacement parameters.

\section{Source of material}

All solvents and starting materials are commercially available and used without further treatment. The title compound was

\footnotetext{
*Corresponding author: Zhen-Qiang Zhang, Henan University of Chinese Medicine, Zhengzhou 450046, P.R. China, e-mail: zhang_zhenqiang@126.com Jun-Ying Song and Zi-Chuang Wang: Henan University of Chinese Medicine, Zhengzhou 450046, P.R. China
}

Table 1: Data collection and handling.

\begin{tabular}{ll}
\hline Crystal: & Yellow cube \\
Size: & $0.30 \times 0.20 \times 0.10 \mathrm{~mm}$ \\
Wavelength: & Cu $K \alpha$ radiation $(1.54184 \AA$ A) \\
$\mu:$ & $0.65 \mathrm{~mm}^{-1}$ \\
Diffractometer, scan mode: & SuperNova, $\omega$ \\
$\theta_{\text {max }}$, completeness: & $71.3^{\circ},>99 \%$ \\
$N(h k l)_{\text {measured }}, N(h k l)_{\text {unique }}, R_{\text {int }}:$ & $11693,5815,0.019$ \\
Criterion for $I_{\text {obs }}, N(h k l)_{\mathrm{gt}}:$ & $I_{\text {obs }}>2 \sigma\left(I_{\text {obs }}\right), 5224$ \\
$N(\text { param })_{\text {refined }}:$ & 430 \\
Programs: & CrysAlis \\
& {$[3,4]$} \\
\hline
\end{tabular}

prepared by a two-step reaction according to the literature [5]. Synthesis of the intermediate rhodamine $B$ hydrazide. To a $50 \mathrm{~mL}$ flask-2-neck, rhodamine B $(1 \mathrm{mmol}, 479 \mathrm{mg})$ was dissolved in $20 \mathrm{~mL}$ ethanol to form a plum purple clear solution. $2.0 \mathrm{~mL}$ hydrazine hydrate ( $80 \%$, excess) was then added dropwise under vigorous stirring at room temperature. And then, the stirred mixture was refluxed for $4 \mathrm{~h}$, during which the solution color changed from dark purple to grass green and finally to light orange. After that, the mixture was cooled and distilled by reduced pressure to yield precipitate. The resulting precipitate was filtered, washed several times with water. Yield: ca. $75 \%$. Synthesis of the title compound quinoxaline-2-carboxaldehyde rhodamine B hydrazone. Rhodamine B hydrazide powder $(0.5 \mathrm{mmol}, 230 \mathrm{mg})$ was dissolved in $15 \mathrm{~mL}$ absolute ethanol. An ethanol solution $15 \mathrm{~mL}$ containing quinoxaline-2-carboxaldehyde $(0.5 \mathrm{mmol}$, $79 \mathrm{mg}$ ) was added to the above rhodamine B hydrazide solution. And then, the stirred mixture was refluxed for $6 \mathrm{~h}$. The reaction solution was reddish brown clarified solution. Then the solvent was concentrated under reduced pressure to yield a yellow precipitate. The precipitate was filtered and washed several times with cold ethanol to obtain yellow powder. Yield: ca. $70 \%$. The yellow powder $(0.02 \mathrm{mmol}$, $11.92 \mathrm{mg}$ ) was dissolved in ethanol/dichloromethane (1:1) $(15 \mathrm{~mL})$ to give a clear yellow solution to evaporate at room temperature. After one weak, pale yellow cube crystals suitable for X-ray diffraction measurements appeared. Yield: ca. $70 \%$. 
Table 2: Fractional atomic coordinates and isotropic or equivalent isotropic displacement parameters $\left(\AA^{2}\right)$.

\begin{tabular}{|c|c|c|c|c|}
\hline Atom & $x$ & $y$ & $z$ & $U_{\text {iso }} * / U_{\text {eq }}$ \\
\hline 01 & $0.57825(10)$ & $0.89454(6)$ & $0.65906(4)$ & $0.0285(2)$ \\
\hline 02 & $0.33937(10)$ & $0.58038(6)$ & $0.49081(4)$ & $0.0298(2)$ \\
\hline N1 & $0.48452(11)$ & $0.77821(7)$ & $0.59775(4)$ & $0.0204(2)$ \\
\hline $\mathrm{N} 2$ & $0.34460(11)$ & $0.80451(7)$ & $0.58256(4)$ & $0.0216(2)$ \\
\hline N3 & $0.08810(12)$ & $0.97492(7)$ & $0.61378(5)$ & $0.0282(2)$ \\
\hline N4 & $-0.07024(12)$ & $0.88213(8)$ & $0.52145(5)$ & $0.0305(3)$ \\
\hline N5 & $0.52290(13)$ & $0.71922(8)$ & $0.32443(5)$ & $0.0306(3)$ \\
\hline N6 & $0.13150(14)$ & $0.41521(9)$ & $0.64134(5)$ & $0.0369(3)$ \\
\hline $\mathrm{C} 1$ & $0.06009(14)$ & $0.85670(9)$ & $0.54116(6)$ & $0.0276(3)$ \\
\hline $\mathrm{H} 1$ & 0.1027 & 0.8045 & 0.5243 & $0.033^{*}$ \\
\hline $\mathrm{C} 2$ & $-0.12768(14)$ & $0.95731(9)$ & $0.54800(6)$ & $0.0291(3)$ \\
\hline C3 & $-0.26626(15)$ & $0.99065(10)$ & $0.52762(7)$ & $0.0364(3)$ \\
\hline H3 & -0.3191 & 0.9610 & 0.4962 & $0.044^{\star}$ \\
\hline $\mathrm{C} 4$ & $-0.32424(16)$ & $1.06584(11)$ & $0.55329(8)$ & $0.0431(4)$ \\
\hline $\mathrm{H} 4$ & -0.4172 & 1.0885 & 0.5394 & $0.052^{*}$ \\
\hline C5 & $-0.24756(18)$ & $1.10972(11)$ & $0.59995(8)$ & $0.0457(4)$ \\
\hline H5 & -0.2897 & 1.1615 & 0.6175 & $0.055^{*}$ \\
\hline $\mathrm{C} 6$ & $-0.11295(17)$ & $1.07919(10)$ & $0.62060(7)$ & $0.0395(3)$ \\
\hline $\mathrm{H} 6$ & -0.0625 & 1.1094 & 0.6524 & $0.047^{*}$ \\
\hline $\mathrm{C} 7$ & $-0.04913(15)$ & $1.00241(9)$ & $0.59430(6)$ & $0.0294(3)$ \\
\hline $\mathrm{C} 8$ & $0.14176(14)$ & $0.90366(9)$ & $0.58694(5)$ & $0.0244(3)$ \\
\hline C9 & $0.29083(14)$ & $0.87616(9)$ & $0.60586(5)$ & $0.0246(3)$ \\
\hline H9 & 0.3464 & 0.9104 & 0.6346 & 0.030 * \\
\hline C10 & $0.59138(13)$ & $0.82226(8)$ & $0.63287(5)$ & $0.0210(2)$ \\
\hline C11 & $0.72108(13)$ & $0.76306(8)$ & 0.63 & $0.0209(2)$ \\
\hline $\mathrm{C} 12$ & $0.85992(14)$ & $0.77886(9)$ & $0.65789(5)$ & $0.0269(3)$ \\
\hline $\mathrm{H} 12$ & 0.8805 & 0.8321 & 0.6809 & $0.032^{\star}$ \\
\hline C13 & $0.96673(14)$ & $0.71423(10)$ & $0.64924(6)$ & $0.0307(3)$ \\
\hline $\mathrm{H} 13$ & 1.0630 & 0.7233 & 0.6661 & $0.037^{\star}$ \\
\hline C14 & $0.93445(14)$ & $0.63582(10)$ & $0.61600(6)$ & $0.0304(3)$ \\
\hline $\mathrm{H} 14$ & 1.0093 & 0.5921 & 0.6107 & $0.036^{*}$ \\
\hline C15 & $9534(14)$ & $52017(9)$ & $0.59039(5)$ & $0.0253(3)$ \\
\hline $\mathrm{H} 15$ & 0.7739 & 0.5663 & 0.5680 & $0.030^{*}$ \\
\hline C16 & 3901(13) & $0.68557(8)$ & $9853(5)$ & $0.0201(2)$ \\
\hline C17 & $3345(12)$ & $0.68888(8)$ & $0.57155(5)$ & $0.0189(2)$ \\
\hline C18 & $0.43751(12)$ & $0.61115(8)$ & $0.58916(5)$ & $0.0197(2)$ \\
\hline C19 & $0.42982(13)$ & $0.58598(9)$ & $0.64700(5)$ & $0.0235(3)$ \\
\hline H19 & 0.4944 & 0.6149 & 0.6753 & $0.028^{*}$ \\
\hline $\mathrm{C} 20$ & $0.33334(14)$ & $0.52138(9)$ & $0.66503(5)$ & $0.0252(3)$ \\
\hline $\mathrm{H} 20$ & 0.3325 & 0.5068 & 0.7050 & 0.030 * \\
\hline $\mathrm{C} 21$ & $0.23514(14)$ & $0.47641(9)$ & $0.62432(6)$ & $0.0253(3)$ \\
\hline $\mathrm{C} 22$ & $0.24610(14)$ & $0.49828(9)$ & $0.56604(5)$ & $0.0261(3)$ \\
\hline $\mathrm{H} 22$ & 0.1855 & 0.4673 & 0.5373 & 0.031 * \\
\hline $\mathrm{C} 23$ & $0.34384(13)$ & $0.56431(8)$ & $0.54948(5)$ & $0.0224(3)$ \\
\hline $\mathrm{C} 24$ & $0.43528(13)$ & $0.64363(8)$ & $0.47032(5)$ & $0.0220(2)$ \\
\hline $\mathrm{C} 25$ & $0.43043(14)$ & $0.65099(9)$ & $0.41051(5)$ & $0.0255(3)$ \\
\hline $\mathrm{H} 25$ & 0.3632 & 0.6144 & 0.3874 & $0.031^{\star}$ \\
\hline $\mathrm{C} 26$ & $0.52363(14)$ & $0.71175(9)$ & $0.38383(5)$ & $0.0248(3)$ \\
\hline $\mathrm{C} 27$ & $0.62020(14)$ & $0.76467(9)$ & $0.42058(6)$ & $0.0261(3)$ \\
\hline $\mathrm{H} 27$ & 0.6843 & 0.073 & 0.4043 & 0.031 * \\
\hline $\mathrm{C} 28$ & $0.62259(13)$ & $0.75531(9)$ & $0.47963(5)$ & $0.0234(3)$ \\
\hline $\mathrm{H} 28$ & 0.6904 & 0.7910 & 0.5031 & $0.028^{*}$ \\
\hline C29 & $0.52999(12)$ & $0.69580(8)$ & $0.50651(5)$ & $0.0196(2)$ \\
\hline $\mathrm{C} 30$ & $0.11270(16)$ & $0.39948(10)$ & $0.70262(6)$ & $0.0326(3)$ \\
\hline $\mathrm{H} 30 \mathrm{~A}$ & 0.2101 & 0.3945 & 0.7236 & 0.039 * \\
\hline
\end{tabular}

Table 2 (continued)

\begin{tabular}{|c|c|c|c|c|}
\hline Atom & $x$ & $y$ & $z$ & $U_{\text {iso }} * / U_{\text {eq }}$ \\
\hline H30B & 0.0616 & 0.3400 & 0.7070 & 0.039 * \\
\hline C31 & $0.02667(18)$ & $0.47554(11)$ & $0.73013(7)$ & $0.0410(4)$ \\
\hline $\mathrm{H} 31 \mathrm{~A}$ & -0.0695 & 0.4814 & 0.7093 & $0.061^{*}$ \\
\hline H31B & 0.0795 & 0.5340 & 0.7281 & $0.061 *$ \\
\hline $\mathrm{H} 31 \mathrm{C}$ & 0.0147 & 0.4601 & 0.7707 & $0.061^{*}$ \\
\hline $\mathrm{C} 32^{\mathrm{a}}$ & $-0.00444(19)$ & $0.39839(12)$ & $0.60262(9)$ & $0.0276(5)$ \\
\hline$H 32 A^{a}$ & -0.0901 & 0.3981 & 0.6262 & $0.033^{*}$ \\
\hline$H 32 B^{a}$ & -0.0174 & 0.4494 & 0.5743 & $0.033^{*}$ \\
\hline $\mathrm{C} 33^{\mathrm{a}}$ & $0.0020(2)$ & $0.30865(15)$ & $0.57076(12)$ & $0.0526(7)$ \\
\hline $\mathrm{H} 33 \mathrm{~A}^{\mathrm{a}}$ & 0.0811 & 0.3109 & 0.5445 & 0.079 * \\
\hline$H 33 B^{a}$ & -0.0910 & 0.2980 & 0.5484 & $0.079^{*}$ \\
\hline $\mathrm{H} 33 \mathrm{C}^{\mathrm{a}}$ & 0.0203 & 0.2583 & 0.5986 & 0.079 * \\
\hline$C 32 A^{b}$ & $0.0807(6)$ & $0.3409(4)$ & $0.5994(3)$ & $0.0272(17)$ \\
\hline $\mathrm{H} 32 \mathrm{C}^{\mathrm{b}}$ & 0.0664 & 0.2818 & 0.6197 & $0.033^{*}$ \\
\hline$H 32 D^{b}$ & 0.1518 & 0.3313 & 0.5698 & $0.033^{*}$ \\
\hline$C 33 A^{b}$ & $-0.0630(8)$ & $0.3776(5)$ & $0.5723(3)$ & $0.040(2)$ \\
\hline$H 33 D^{b}$ & -0.1277 & 0.3925 & 0.6028 & $0.059^{\star}$ \\
\hline $\mathrm{H} 33 \mathrm{E}^{\mathrm{b}}$ & -0.1091 & 0.3308 & 0.5466 & $0.059^{*}$ \\
\hline $\mathrm{H} 33 \mathrm{~F}^{\mathrm{b}}$ & -0.0449 & 0.4334 & 0.5500 & 0.059 * \\
\hline C34 & $0.42692(16)$ & $0.66339(11)$ & $0.28591(6)$ & $0.0347(3)$ \\
\hline $\mathrm{H} 34 \mathrm{~A}$ & 0.4109 & 0.6034 & 0.3048 & $0.042^{\star}$ \\
\hline H34B & 0.4765 & 0.6510 & 0.2502 & $0.042^{*}$ \\
\hline C35 & $0.28000(19)$ & $0.70694(13)$ & $0.26948(8)$ & $0.0517(4)$ \\
\hline $\mathrm{H} 35 \mathrm{~A}$ & 0.2214 & 0.6650 & 0.2441 & $0.077^{*}$ \\
\hline H35B & 0.2942 & 0.7651 & 0.2491 & $0.077^{\star}$ \\
\hline $\mathrm{H} 35 \mathrm{C}$ & 0.2293 & 0.7189 & 0.3045 & $0.077^{\star}$ \\
\hline C36 & $0.61503(17)$ & $0.78699(10)$ & $0.29814(6)$ & $0.0351(3)$ \\
\hline $\mathrm{H} 36 \mathrm{~A}$ & 0.6213 & 0.7708 & 0.2569 & $0.042^{\star}$ \\
\hline H36B & 0.7147 & 0.7830 & 0.3172 & $0.042^{*}$ \\
\hline C37 & $0.56214(19)$ & $0.88604(11)$ & $0.30189(7)$ & $0.0428(4)$ \\
\hline $\mathrm{H} 37 \mathrm{~A}$ & 0.4654 & 0.8917 & 0.2815 & $0.064^{*}$ \\
\hline H37B & 0.6306 & 0.9272 & 0.2840 & $0.064^{\star}$ \\
\hline $\mathrm{H} 37 \mathrm{C}$ & 0.5563 & 0.9031 & 0.3426 & $0.064^{\star}$ \\
\hline
\end{tabular}

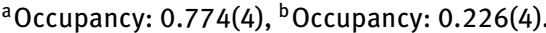

\section{Experimental details}

The $\mathrm{H}$ atoms were added geometrically using riding models and refined isotropically.

\section{Comment}

Quinoxalines are well known fluorescent compounds known for high quantum yields and have attracted much attention due to their potential functions in molecular probes and in polymer fields $[6,7]$. Quinoxalines are easy to prepare and numerous derivatives have been designed as biologically active materials [8]. Rhodamine B derivatives have attracted attention by virtue of their long-wavelength emission and availability $[9,10]$. In this paper, we report a quinoxaline-2carboxaldehyde rhodamine B hydrazone compound. The title compound is the potential fluorescent probe for certain metal ion.

The asymmetric unit contains a neutral molecule in a ring-closed form. The amide $\mathrm{C}=\mathrm{O}$ bond distance is 
1.2216(15) $\AA$, indicative of the keto form. The dihedral angle between xanthene least-square plane and quinoxaline aroylhydrozone group is $88.6^{\circ}$. The thermal vibration of C32 and $\mathrm{C} 33$ atoms at the end of xanthene is larger, so they have been disordered treated. There are apparent $\pi-\pi$ contacts between adjacent quinoxaline groups of the adjacent molecules. The quinoxaline groups of the adjacent molecules are parallel with the face-to-face distances of $3.305 \AA$. There are also $\pi$ $\pi$ contacts between adjacent xanthene groups of the adjacent molecules. The face-to-face distances of parallel plane of adjacent molecules is $3.569 \AA$ And $3.553 \AA$ A. The relatively short distances and the parallel xanthene planes indicate the presence of strong intermolecular $\pi-\pi$ contacts. All geometric parameters are in the expected ranges [11, 12].

Acknowledgements: This work was supported by the Scientific and Technological Brainstorm Project of Henan Province (192102310214) and the Foundation of Henan Educational Committee (19A520005, 19A360021).

\section{References}

1. Rigaku Oxford Diffraction. CrysAlis Pro Software system, version 1. 171. 38. 43f. Rigaku Oxford Diffraction (2015).

2. Dolomanov, O. V.; Bourhis, L. J.; Gildea, R. J.; Howard, J. A. K.; Puschmann, H.: OLEX2: a complete structure solution, refinement and analysis program. J. Appl. Crystallogr. 42 (2009) 339-341.

3. Sheldrick, G. M.: Crystal structure refinement with SHELXL. Acta Crystallogr. C71 (2015) 3-8.
4. Sheldrick, G. M.: SHELXT-integrated space-group and crystal-structure determination. Acta Crystallogr. A71 (2015) 3-8.

5. Xiang, Y.; Tong, A.; Jin, P.; Ju, Y.: New fluorescent rhodamine hydrazone chemosensor for $\mathrm{Cu}(\mathrm{II})$ with high selectivity and sensitivity. Org. Lett. 8 (2006) 2863-2866.

6. Kumar, A.; Kumar, V.; Diwan, U.; Upadhyay, K. K.: Highly sensitive and selective naked-eye detection of $\mathrm{Cu}^{2+}$ in aqueous medium by a ninhydrin-quinoxaline derivative. Sens. Actuators, B. 176 (2013) 420-427.

7. Goswami, S.; Chakraborty, S.; Das, A. K.; Manna, A.; Bhattacharyya, A.; Quah, C. K.; Fun H.-K.: Selective colorimetric and ratiometric probe for $\mathrm{Ni}(\mathrm{II})$ in quinoxaline matrix with the single crystal X-ray structure. RSC Adv. 4 (2014) 20922-20926.

8. Lo, K. K.-W.; Tsang, K. H.-K.; Sze, K.-S.: Utilization of the highly environment-sensitive emission properties of rhenium(I) amidodipyridoquinoxaline biotin complexes in the development of biological probes. Inorg. Chem. 45 (2006) 1714-1722.

9. Kim, H. N.; Lee, M. H.; Kim, H. J.; Kim, J. S.; Yoon, J.: A new trend in rhodamine-based chemosensors: application of spirolactam ring-opening to sensing ions. Chem. Soc. Rev. 37 (2008) 1465-1472.

10. Kwon, J. Y.; Jang, Y. J.; Lee, Y. J.; Kim, K. M.; Seo, M. S.; Nam, W. W.; Yoon, J. Y.: A highly selective fluorescent chemosensor for $\mathrm{Pb}^{2+}$. J. Am. Chem. Soc. 127 (2005) 10107-10111.

11. Yuan, J.; Cheng, D.: Crystal structure of (E)-3',6'bis(ethylamino)-2',7'-dimethyl-2-((pyridin-2-ylmethylene) amino)spiro[isoindoline-1,9'-xanthen]-3-one, $\mathrm{C}_{32} \mathrm{H}_{31} \mathrm{~N}_{5} \mathrm{O}_{2}$. Z . Kristallogr. NCS 234 (2019) 149-151.

12. Xing, A.-P.; Zeng, D.; Wang, X.-L.: Crystal structure of (E)-2-(((6bromopyridin-2-yl)methylene)amino)-3',6'-bis(diethylamino) spiro[isoindoline-1,9'-xanthen]-3-one, $\mathrm{C}_{34} \mathrm{H}_{34} \mathrm{~N}_{5} \mathrm{O}_{2} \mathrm{Br}$. Z. Kristallogr. NCS 234 (2019) 309-311. 